\title{
Conceitos disciplinares em uso por estudantes de licenciatura e de mestrado em Enfermagem
}

\author{
Disciplinary concepts in use by undergraduate and master's students in Nursing \\ Conceptos disciplinarios en uso por los estudiantes de grado y máster en Enfermeira \\ Paulo Joaquim Pina Queirós*
}

\section{Resumo}

Enquadramento: A utilização da linguagem disciplinar, de conceitos, faz parte da integração na comunidade científica.

Objetivos: Diagnóstico da situação quanto aos termos usados para definir enfermagem; o conjunto de conceitos em uso e o seu alinhamento com conceitos centrais e padrões de conhecimento, com vista a direcionar a formação em maior consonância com o estado da arte da teoria.

Metodologia: Estudo descritivo, com análise de conteúdo, em duas amostras de estudantes de licenciatura e de mestrado.

Resultados: Encontrámos dispersão de termos e conceitos a revelar dificuldade na definição de enfermagem; o cuidar é o termo e conceito com maior utilização; os conceitos de transição e bem-estar não estão apropriados pelos estudantes a iniciar o mestrado; os estudantes da licenciatura não dão centralidade ao termo transição mas valorizam com relevância o bem-estar; ambos facilmente percecionam a enfermagem como ciência, valorizando o padrão empírico; os estudantes a iniciar o mestrado, por afastados do ensino formal há mais tempo, apresentam uma menor apropriação de termos e conceitos disciplinares propostos pela teoria de enfermagem. Conclusão: Torna-se relevante que a formação inicial, pós-graduada e a contínua, contribua e beneficie da sistematização da teoria produzida e expressa em linguagem disciplinar específica.

Palavras-chave: teoria de enfermagem; educação em enfermagem.

\begin{abstract}
Background: The use of a disciplinary language and concepts is part of the integration into the scientific community.

Objectives: To identify the terms used to define nursing, the set of concepts in use and its alignment with core concepts and patterns of knowing so that education is more in line with the state of the art of the theory.

Methodology: Descriptive study with content analysis using two samples of undergraduate and master's students.

Results: The dispersion of terms and concepts showed difficulties in defining nursing; the term and concept of caring was used more often; the concepts of transition and well-being were not used by beginning master's students; the term of transition was not central to undergraduates, but they greatly valued wellbeing; both undergraduate and master's students easily perceived nursing as a science, emphasising the empirical pattern of knowledge; beginning master's students, by being absent from formal education for a longer period, used disciplinary terms and concepts proposed by the nursing theory less often.

Conclusion: Initial, postgraduate and lifelong training should contribute to and benefit from the systematisation of the theory produced and expressed in specific disciplinary language.
\end{abstract}

Keywords: nursing theory; nursing education.

\footnotetext{
* Doutoramento em Desenvolvimento e Intervenção Psicológica. Mestre em Saúde Ocupacional. Licenciatura em História e em Enfermagem de Reabilitação. Pósdoutorando ICBAS-UP. Professor Coordenador, Escola Superior de Enfermagem de Coimbra, 3046-851, Coimbra, Portugal [pauloqueiros@esenfc.pt]. Morada para correspondência: Rua do Açude, $n^{\circ}$ 150, 3020-263, Coimbra, Portugal
}

\section{Resumen}

Marco contextual: El uso del lenguaje disciplinario de conceptos forma parte de la integración en la comunidad científica.

Objetivo: Diagnosticar la situación con respecto a los términos utilizados para definir la enfermería, el conjunto de conceptos en uso y su alineación con los conceptos centrales y los patrones de conocimiento, con el fin de que la formación esté en consonancia con los últimos avances científicos de la teoría.

Metodología: Se trata de un estudio descriptivo, con análisis de contenido, en dos muestras de estudiantes de grado y máster.

Resultados: Así, encontramos una dispersión de términos y conceptos que muestra la dificultad de definir la enfermería. El término y el concepto más usado es atender; los conceptos de transición y bienestar no son apropiados para los estudiantes que comienzan el máster; los estudiantes de grado no dan primacía al término transición, pero sí un valor relevante al bienestar; ambos entienden la enfermería como ciencia y valoran el patrón empírico, y los estudiantes que van a empezar el máster, por llevar más tiempo desconectados de la educación formal, presentan una asignación más baja de términos y conceptos disciplinarios propuestos por la teoría de enfermería.

Conclusión: Se considera relevante que la formación inicial, de posgrado y continua, pueda aportar y beneficiarse de la sistematización de la teoría producida y expresada en el lenguaje disciplinario específico.

Palabras clave: teoría de enfermería; educación en enfermería.

Recebido para publicação em: 27.05.13

Aceite para publicação em: 28.02 .14 


\section{Introduçáo}

As disciplinas têm uma linguagem própria, uma estrutura de comunicação dos seus fenómenos de interesse e da sua interpretação do real que se expressa por termos e conceitos. Alguns conceitos pelo seu poder interpretativo, explicativo e simbólico adquirem centralidade no âmbito da disciplina; e tornam-se elementos de precisão do núcleo de interesse, da perspetiva peculiar, essenciais para a identificação da matriz disciplinar.

"Uma disciplina científica é determinada por uma organização mental. É aquilo a que se chama, em filosofia das ciências, uma matriz disciplinar ou um paradigma, ou seja, uma estrutura mental, consciente ou não, que serve para classificar o mundo a fim de poder abordá-lo" (Fourez, 2008, p. 111). É neste entendimento que, enquanto linguagem trabalhada, sintetizada, reconhecida e com significado num universo específico, comunidade científica, permitindo leituras narradas da realidade e a sua sistematização, dão corpo a uma "série de pressupostos, de normas, de instrumentos, de maneiras de ver, ... dão a sua fisionomia ao saber que essa disciplina estrutura" (Fourez, 2008, p. 111).

Hoje, tem-se uma ideia dinâmica dos conceitos, que devem reportar-se ao seu uso específico mas contextualizado. "Os teóricos agora entendem que o significado conceitual é criado pelos estudiosos para auxiliar a transmitir seu significado aos leitores" (McEwen \& Wills, 2009, p. 76). A apropriação de conceitos e a utilização da linguagem disciplinar faz parte do processo pelo qual os neófitos, estudantes ou profissionais, se integram na comunidade científica, criando e recriando conhecimento em ação, tendo por base uma racionalidade prático-reflexiva (Medina, 1999).

Propomo-nos neste estudo, junto de estudantes de licenciatura e a iniciar o mestrado em enfermagem, identificar os termos usados para a definição de enfermagem; enumerar os conceitos que apontam como específicos ou com significado específico na enfermagem; ter uma visão de conjunto dos termos e conceitos que usam; descrever os termos e conceitos que estão em alinhamento com os conceitos centrais e os padrões de conhecimento em enfermagem definidos na literatura de enfermagem.

\section{Enquadramento}

Os conceitos desempenham um papel estruturante nas disciplinas. É através dos conceitos que se definem os núcleos centrais, se cria e enriquece a linguagem disciplinar. Os conceitos comportam uma dupla função, focalizar aquilo que é o objetivo de determinado corpo de saberes, a perspetiva própria sobre determinado real, e proporcionar narrativas construídas no âmbito dessa singular interpretação. "As narrativas ligadas ao conceito dão às pessoas «palavras para dizê-lo». As palavras para dizer a sua experiência" (Fourez, 2008, p. 276).

Os conceitos, sendo formulações mentais ou ideias usadas para representar as experiências, "são formulados em palavras que possibilitam que as pessoas comuniquem o significado das realidades no mundo" e assim "explicam a matéria das teorias de uma disciplina” (McEwen \& Wills, 2009, p. 76).

A ciência é o estabelecimento de uma linguagem com vista a uma economia de pensamento e de comunicação, "uma gigantesca operação de estandartização de saberes, de grelhas de leitura, de noções, de procedimentos, de valores, etc. As matrizes disciplinares podem ser vistas com este tipo de normalização" (Fourez, 2008, p. 127). Os conceitos podem ser próprios, emergindo da disciplina, mas "também se 'propagam' ... de uma disciplina para outra, fortalecendo pontos de vista novos..." (Fourez, 2008, p. 119).

Yura e Torres de forma pioneira em 1975 propuseram para conceitos focais na disciplina, a enfermagem, a pessoa (man), a sociedade, e a saúde. McEwen e Wills (2009, p.67) referem que "Fawcett escreveu, pela primeiravez,sobre os conceitos centrais de enfermagem em 1978 e formalizou-os como metaparadigma de enfermagem em 1984". Fawcett em 2005 mantém os conceitos: enfermagem, saúde, ambiente (que anteriormente já tinha derivado de sociedade) e o ser humano (que evoluiu do conceito pessoa). $\mathrm{O}$ Conselho de Enfermagem da Ordem dos Enfermeiros, em 2002, utiliza os conceitos saúde, pessoas, ambiente e cuidados de enfermagem, para o enquadramento concetual dos cuidados de enfermagem.

A aceitação de indivíduo, saúde, ambiente, enfermagem como um metaparadigma de enfermagem não reúne consenso (McEwen \& Willis, 2009). Newman, em 1983, apontou como principais componentes da disciplina "enfermagem (como 
uma ação), cliente (ser humano), meio ambiente (do cliente e do enfermeiro-cliente), e a saúde" (p.389). E Kim (2010) identificou uma tipologia e estrutura organizadora com quatro domínios: paciente, paciente-enfermeiro, prática e ambiente. Em 1994, Meleis e Trangenstein, ao afirmarem que a enfermagem consiste na facilitação dos processos de transição, no sentido de se alcançar uma maior sensação de bem-estar transportam, a nosso ver, para uma centralidade concetual os termos facilitação, transição e bem-estar. Meleis (2012) sintetiza como sete conceitos centrais: interação, paciente de enfermagem, transições, processo de enfermagem, ambiente, terapêutica de enfermagem e saúde.

A enfermagem como possuí características das ciências sociais e comportamentais, bem como das ciências biológicas, deve contar com múltiplas formas de conhecimento (McEwen \& Wills, 2009), ou seja, diversificados padrões de conhecimento. A epistemologia da enfermagem preocupa-se com "o estudo das origens do conhecimento de enfermagem, as suas estruturas e os seus métodos, os padrões de conhecimento dos seus membros e os critérios para a validação das afirmações do conhecimento" (Schultz \& Meleis, 1988, p.21).

A partir do trabalho seminal de Carper (2006) identificando os padrões empírico, estético, pessoal e ético, como fundamentais para o conhecimento em enfermagem, vários autores, com similitudes e dessemelhanças, propõem acrescentos e outras formulações. É o caso dos padrões clínico e conceitual (Schultz \& Meleis, 1988); experimental, interpessoal e intuitivo (Moch,1990); contexto (White, 2006); processual, relacional, cultural e tácito (Abreu, 2008). "A enfermagem é dotada tanto do conhecimento científico como do conhecimento que pode ser denominado sabedoria convencional (aquele que não é empiricamente testado)" (McEwen \& Wills, 2009, p. 37). Ou, como afirma Kim (2010), "conhecimento público" e "conhecimento privado". O padrão do conhecimento empírico dá forma ao conhecimento público; outros padrões de conhecimento como estético, ético, pessoal, clínico, conceitual, interpessoal, intuitivo, contextual, processual, relacional, cultural e tácito, estruturam-se na forma de conhecimento privado. Ambas as formas, identitárias do conhecer em enfermagem, articulam-se em espiral, num movimento de constante translação, entre investigação/teorização e ação na prática clínica.
Construindo conhecimento a partir da reflexão sobre as suas práticas como profissionais "prático-reflexivos cuja ação se baseia num conhecimento prático e tácito que se ativa durante a ação e no qual podem, sob o ponto de vista heurístico, distinguir-se três componentes: conhecimento em ação, reflexão em ação e reflexão sobre a reflexão em ação" (Moya, 2005, p.487-490). Sendo, como refere Waldow (2009), a enfermagem uma actividade essencialmente prática, o processo de cuidar prevê no momento de cuidar a reflexão na ação.

\section{Metodologia}

Estudo qualitativo, de natureza descritiva, com análise de conteúdo inspirada em Bardin (1995), em que se analisaram respostas a perguntas simples e diretas, provenientes de duas amostras de conveniência, respondendo à pergunta de investigação como definem enfermagem e quais os conceitos e termos usados?. O processo de codificação das respostas seguiu a técnica de recorte com registo de unidades base (de registo) com análise de unidades repetidas, similares, ambíguas e de grupo, de enumeração com a determinação de frequências relativas e absolutas, e a agregação em categorias (sem a existência de categorias a priori).

A um grupo de estudantes a iniciar a unidade curricular de Teoria de Enfermagem do curso de mestrado em Enfermagem Médico-Cirúrgica, no ano letivo 2012-2013, de uma escola superior de enfermagem, solicitou-se que respondessem à seguinte questão: 0 que é a enfermagem? Na mesma escola, a estudantes do $4^{\circ}$ ano da licenciatura em Enfermagem, a iniciar a frequência da unidade curricular de opção Gestão do Autocuidado Terapêutico, pediu-se, num primeiro momento, que respondessem à mesma pergunta, e em sessão posterior, convidou-se a enumerar os conceitos que entendam como específicos ou com significado específico na enfermagem. Do conjunto destas duas amostras de informantes (grupo I e II) resultaram três séries de dados, identificadas respetivamente neste estudo por I A, II B e II C (as duas primeiras relativas aos termos extraídos das definições de enfermagem em cada grupo, e a última referente aos conceitos entendidos como específicos ou com significado específico apontados pelo grupo II). As definições produzidas nas séries A e B foram 
analisadas, com extração dos termos utilizados para a definição do que é a enfermagem e agrupados por ordem decrescente de frequência. Na série $C$ procedeu-se de igual forma em relação aos conceitos evocados pelos estudantes. Efetuou-se ainda uma análise conjunta das três séries e a enumeração por ordem decrescente de frequência. Em todas as séries determinaram-se índices de referência. Por fim, procedeu-se à identificação dos termos e conceitos referidos nas séries com os conceitos centrais e com os padrões de conhecimento em enfermagem da literatura. Todos os participantes foram informados dos objetivos deste estudo e a todos foi pedida autorização para a utilização dos dados, mantendo o anonimato. O grupo de estudantes a iniciar 0 Mestrado é constituído por 26 enfermeiros, com um média de idades de 30,65 anos, num intervalo entre
22 e 42, média de 8,27 anos de serviço. 0 grupo de estudantes da licenciatura é constituído por 24 indivíduos com idades entre os 21 e 39, com uma média de 22,5 anos. Neste estudo, para o material (palavras) extraído das definições de enfermagem utilizaremos a designação de termo e reservamos a designação de conceito para o material sintetizado da pergunta concreta sobre conceitos entendidos como específicos ou com significado específico.

\section{Resultados}

Os enfermeiros a iniciar o Mestrado (grupo I) produziram definições de enfermagem das quais foram extraídos 66 termos com um total de 221 referências (Tabela 1).

Tabela1

Termos utilizados e sua frequência na definição de enfermagem pelos estudantes de mestrado

\begin{tabular}{|c|c|c|c|c|c|}
\hline cuidar & 15 & Neces.humanas básicas & 3 & curativo & 1 \\
\hline científico & 14 & psicológico & 3 & desenv. de competências & 1 \\
\hline pessoa & 12 & relação de ajuda/ empática & 3 & emocional & 1 \\
\hline saúde & 12 & saber ser, estar, fazer & 3 & físico & 1 \\
\hline profissão & 10 & substituir o doente & 3 & fisiologia & 1 \\
\hline disciplina & 9 & etapa de vida & 2 & grupos & 1 \\
\hline promover & 9 & focos & 2 & interação & 1 \\
\hline arte & 8 & hábitos de vida saudável & 2 & interdisciplinaridade & 1 \\
\hline doença & 8 & objetivos & 2 & meio ambiente & 1 \\
\hline ciclo de vida & 7 & recolha de dados & 2 & meio social e cultural & 1 \\
\hline técnico/tecnologia & 7 & tratamento & 2 & método de trabalho & 1 \\
\hline conhecimentos & 6 & independência & 2 & multidisciplinaridade & 1 \\
\hline holístico & 6 & activ.interdependentes & 1 & olhar e ver & 1 \\
\hline individuo & 6 & ajuda ao próximo & 1 & orientativo & 1 \\
\hline prevenção & 6 & angústia & 1 & potencial & 1 \\
\hline ensino/educação p/saúde & 5 & aprender habilidades & 1 & qualidade de vida & 1 \\
\hline família & 5 & autenticidade profissional & 1 & queixa & 1 \\
\hline avaliação & 3 & biopsicocultural & 1 & recuperação & 1 \\
\hline bem-estar & 3 & cliente & 1 & reinserção social & 1 \\
\hline diagnóstico de enfermag. & 3 & comunidade & 1 & ser biopsicossocial & 1 \\
\hline humano & 3 & continuo saúde-doença & 1 & situação de vida & 1 \\
\hline \multirow[t]{2}{*}{ intervenções } & 3 & curador & 1 & social & 1 \\
\hline & & & & TOTAL & 221 \\
\hline
\end{tabular}

Não deixa de ser expressiva a escolha destes termos para definir a enfermagem, sendo de realçar também a relativa dispersão dos mesmos. Encontrámos 32 termos, quase metade, referidos apenas com uma referência, e sete termos com duas referências. 


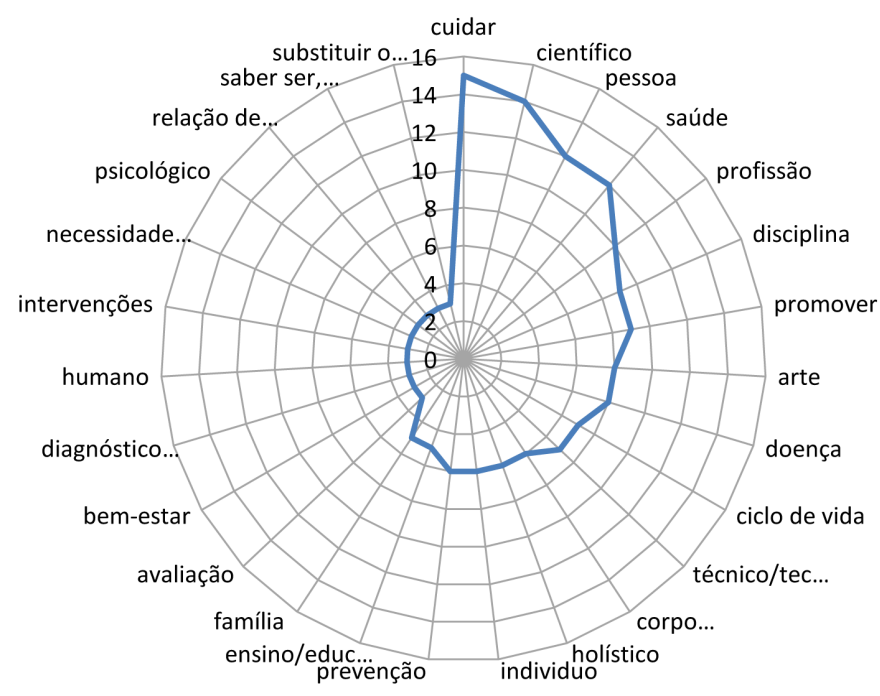

Figura 1. Termos com frequências mais elevadas na definição de enfermagem pelos estudantes de mestrado

As frequências revelam como termos mais usados: cuidar (15); científico (14); pessoa (12); saúde (12); profissão (10); disciplina (9); promover (9); arte (8); doença (8); ciclo de vida (7); técnico/tecnologia (7) (Figura 1).

Os estudantes do $4^{0}$ ano do Curso de Licenciatura em Enfermagem a frequentar a unidade curricular de opção Gestão do Autocuidado Terapêutico (amostra II série B) definiram enfermagem utilizando 100 termos, num total de 309 referências. Os estudantes desta amostra definem a enfermagem com uma maior dispersão terminológica $(309 / 24=12,87)$ do que os da amostra anterior $(221 / 26=8,5)$. Os termos cimeiros, com frequências mais altas, são: saúde (17); cuidar (16); doente (16); pessoa (16); ciência (14); objetivo (13); humano (10); transição (8); bemestar (7); promover (7). Outros termos, como sejam: arte, ajuda e processos apresentam 6 referências; autocuidado e profissão 5; ciclo vital e facilitar 4; cultura, disciplina e indivíduo 3; ação e contexto 2. A lista termina em grande dispersão, 51 termos com apenas uma referência (Tabela 2).

\section{Tabela 2}

Termos utilizados e sua frequência na definição de enfermagem pelos estudantes de licenciatura

\begin{tabular}{|c|c|c|c|c|c|}
\hline saúde & 17 & psicológico & 3 & família & 1 \\
\hline cuidar & 16 & teóricos & 3 & fases & 1 \\
\hline doente & 16 & ação & 2 & fomentar & 1 \\
\hline pessoa & 16 & apoiar & 2 & formação & 1 \\
\hline ciência & 14 & autonomia & 2 & funcional & 1 \\
\hline objetivo & 13 & capacidade & 2 & fundamental & 1 \\
\hline humano & 10 & complexa & 2 & gerais & 1 \\
\hline transição & 8 & contexto & 2 & grupo & 1 \\
\hline bem-estar & 7 & história & 2 & guiar & 1 \\
\hline promover & 7 & holística & 2 & inata & 1 \\
\hline ajuda & 6 & motivação & 2 & incapacidade & 1 \\
\hline arte & 6 & necessidades & 2 & influenciar & 1 \\
\hline prática & 6 & qualidades & 2 & intervir & 1 \\
\hline processos & 6 & substituir & 2 & máximo & 1 \\
\hline ser & 6 & vivências & 2 & meio & 1 \\
\hline Independência/dependência & 6 & académica & 1 & modelo & 1 \\
\hline \multirow[t]{2}{*}{ autocuidado } & 5 & alcançar & 1 & monetárias & 1 \\
\hline & PAULO JOAC & JuEIRÓS & rma & ncia-IV-n.' 2-2014 & \\
\hline
\end{tabular}




\begin{tabular}{|c|c|c|c|c|c|}
\hline profissão & 5 & alteradas & 1 & natural & 1 \\
\hline satisfação & 5 & ao longo da vida & 1 & outro & 1 \\
\hline actividades da vida diária & 4 & aptidão & 1 & papel & 1 \\
\hline biopsicossocial/cultural & 4 & assistir & 1 & pensamento & 1 \\
\hline ciclo vital & 4 & atingir & 1 & procedimentos & 1 \\
\hline facilitar & 4 & auxílio & 1 & realização & 1 \\
\hline prestar & 4 & básicas & 1 & reconhecimento & 1 \\
\hline social & 4 & componentes & 1 & recuperação & 1 \\
\hline áreas & 3 & comunidade & 1 & religião & 1 \\
\hline cultura & 3 & condições & 1 & respostas & 1 \\
\hline disciplina & 3 & conhecimentos & 1 & saber & 1 \\
\hline estudo & 3 & conjunto & 1 & todo & 1 \\
\hline físico & 3 & crenças & 1 & tratar & 1 \\
\hline foco & 3 & de si & 1 & vertentes & 1 \\
\hline individualizada & 3 & encontros & 1 & vida & 1 \\
\hline individuo & 3 & ensino & 1 & Total & 309 \\
\hline prevenção & 3 & execução & 1 & & \\
\hline
\end{tabular}

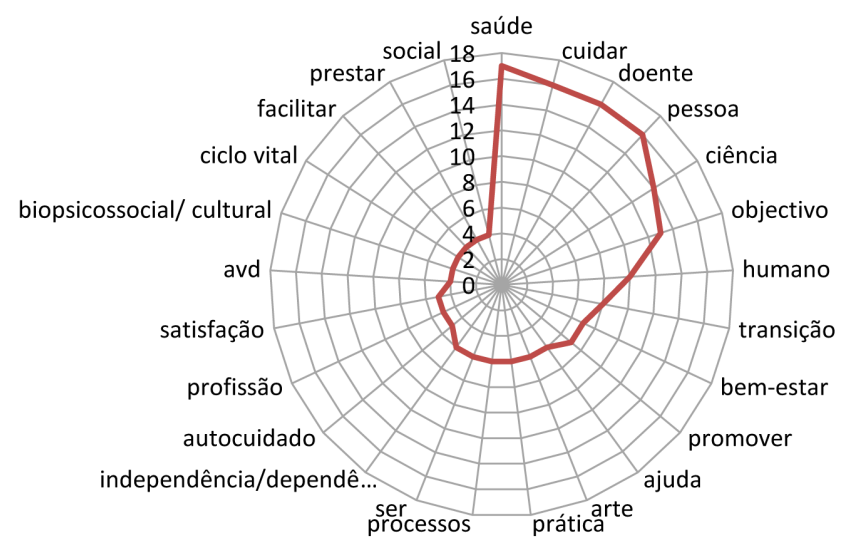

Figura 2. Termos com frequências mais elevadas na definição de enfermagem pelos estudantes de licenciatura

A ordenação dos termos por ordem decrescente de referências permite percecionar que a enfermagem é definida com recurso aos termos saúde, cuidar, doente, pessoa, ciência, objetivo, humano, transição, bem-estar, promover, ajuda, arte, ... (Figura 2).

Os estudantes do $4^{\circ}$ ano (amostra II, série C) identificaram 75 conceitos definidores de enfermagem, específicos ou com significado específico na disciplina, para um total de 248 conceitos expressos. O índice de conceitos 248/24 $(10,33)$ é inferior ao índice dos termos extraídos nas definições de enfermagem 309/24 (12,87), nesse sentido as definições produzidas comportam maior riqueza conceptual que os conceitos evocados, estes por natureza mais sintéticos. 


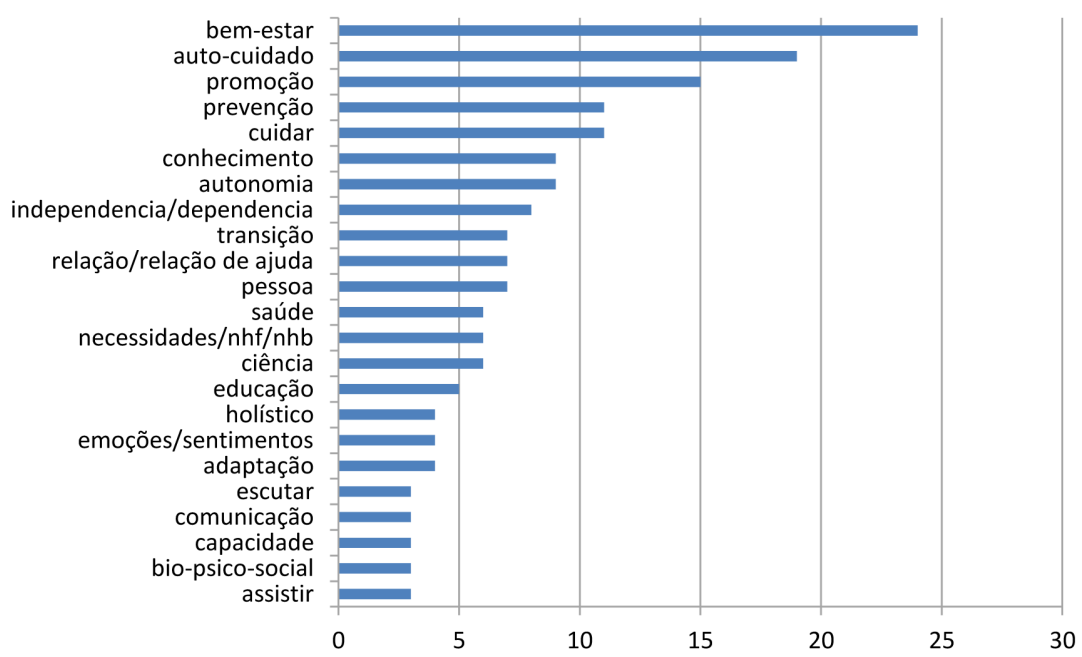

Figura 3. Conceitos, específicos ou com significado específico na enfermagem com frequências mais elevadas, apontados pelos estudantes de licenciatura e sua frequência

Os conceitos com frequências mais elevadas são: bem-estar (24); autocuidado (19); promoção (15); cuidar (11); prevenção (11); autonomia (9); conhecimento (9); independência/dependência (8); pessoa (7); relação/relação de ajuda (7); transição (7). Seguem-se os conceitos ciência, necessidades, saúde, com uma frequência de 6; educação com 5; referidos por 4 estudantes os conceitos adaptação, emoções/ sentimentos, holístico; e com uma frequência de 3 , assistir, bio-psico-social, capacidade, comunicação, escutar (Figura 3).

Outros conceitos que reuniram duas referências são: adesão terapêutica; arte; atividades da vida diária; cuidados continuados; cuidados paliativos; diagnósticos; doença; empatia; ensinos; família; formação; funcionalidade; intervenções; investigação; motivação; potencialização; salutogénese; transculturalidade; vontade.

Os conceitos referenciados uma vez foram: ajuda; avaliação; comportamento; continuidade; criatividade; disciplina; equipa; facilitar; fenómeno; filosofia; foco; gestão; humanização; igualdade; monitorização; pensamento crítico; plano de cuidados; prescrever; pró-ativo; processo; profissão; reabilitação; recuperação; reflexão; responsabilidade; satisfação; tomada de decisão; transação; treinar.

A sequência dos termos mais utilizados pelos estudantes de Mestrado (I A) na definição de enfermagem foi: cuidar, científico/ciência, pessoas, saúde, profissão, disciplina, promover, arte, doença. A sequência dos estudantes da licenciatura (II B) fica ordenada da seguinte forma: saúde, cuidar, doente, pessoa, ciência, objetivo, humano, transição, bem-estar, promover. Em comum e a ocupar os primeiros lugares temos: cuidar, científico/ciência, pessoa, saúde. Os termos autonomia, transição e autocuidado não são referidos pelos estudantes de Mestrado. Todos os outros quinze termos são referenciados pelos estudantes de Mestrado e de Licenciatura.

Quando se junta à comparação os conceitos com especificidade ou significado próprio na disciplina, evocados pelos estudantes da licenciatura, verificamos que apenas não é referido o termo objetivo.

Os conceitos mais referidos (II C) são: bem-estar, autocuidado, promover, prevenção e cuidar. Em relação aos quatro primeiros lugares de termos mais referidos, são comuns nas séries I A e II B: cuidar, científico/ciência, pessoa e saúde. Mas destes só o cuidar é referido, nos primeiros lugares, como conceito específico (em II C). Os outros descem para sétimo e oitavo lugar de referência. Em sentido inverso, os conceitos de bem-estar, autocuidado, que ocupam os dois primeiros lugares na série II C, são apenas referidos em posições inferiores nas outras duas séries: o bem-estar $10^{\circ}$ lugar na I A e $7^{\mathrm{a}}$ na II B, e o autocuidado em $9^{\circ}$ lugar na série II B (Tabela 3). 
Tabela 3

Termos e conceitos utilizados e apontados pelos estudantes de licenciatura e de mestrado, lugar de ordem decrescente e frequências

\begin{tabular}{lcccccc}
\hline Amostra/ séries & \multicolumn{2}{c}{ I A } & \multicolumn{2}{c}{ II } & \multicolumn{2}{c}{ II C } \\
\hline Cuidar & $1^{\circ}$ & 15 & $2^{\circ}$ & 16 & $4^{\circ}$ & 11 \\
Científico/ciência & $2^{\circ}$ & 14 & $3^{\circ}$ & 14 & $\left(8^{\circ}\right.$ & $6)$ \\
Pessoa & $3^{\circ}$ & 12 & $2^{\circ}$ & 16 & $\left(7^{\circ}\right.$ & $7)$ \\
Saúde & $3^{\circ}$ & 12 & $1^{\circ}$ & 17 & $\left(8^{\circ}\right.$ & $6)$ \\
Profissão & $4^{\circ}$ & 10 & $\left(9^{\circ}\right.$ & $5)$ & $\left(13^{\circ}\right.$ & $1)$ \\
Disciplina & $5^{\circ}$ & 9 & $\left(11^{\circ}\right.$ & $3)$ & $\left(13^{\circ}\right.$ & $1)$ \\
Promover & $5^{\circ}$ & 9 & $\left(7^{\circ}\right.$ & $7)$ & $3^{\circ}$ & 15 \\
Arte & $6^{\circ}$ & 8 & $\left(8^{\circ}\right.$ & $6)$ & $\left(11^{\circ}\right.$ & $2)$ \\
Doença & $6^{\circ}$ & 8 & $2^{\circ}$ & 16 & $\left(12^{\circ}\right.$ & $2)$ \\
Objetivos & $\left(11^{\circ}\right.$ & $2)$ & $4^{\circ}$ & 13 & & \\
Independente/dependente & $\left(11^{\circ}\right.$ & $2)$ & $\left(8^{\circ}\right.$ & $6)$ & $6^{\circ}$ & 8 \\
Conhecimento & $\left(8^{\circ}\right.$ & $6)$ & $\left(13^{\circ}\right.$ & $1)$ & $5^{\circ}$ & 9 \\
Autonomia & & & $\left(12^{\circ}\right.$ & $2)$ & $5^{\circ}$ & 9 \\
Prevenção & $\left(8^{\circ}\right.$ & $6)$ & $\left(11^{\circ}\right.$ & $3)$ & $4^{\circ}$ & 11 \\
Humano & $\left(10^{\circ}\right.$ & $3)$ & $5^{\circ}$ & 10 & $\left(13^{\circ}\right.$ & $1)$ \\
Transição & & & $6^{\circ}$ & 8 & $\left(7^{\circ}\right.$ & $7)$ \\
Bem-estar & $\left(10^{\circ}\right.$ & $3)$ & $\left(7^{\circ}\right.$ & $7)$ & $1^{\circ}$ & 24 \\
Autocuidado & & & $\left(9^{\circ}\right.$ & $5)$ & $2^{\circ}$ & 19 \\
\hline
\end{tabular}

O somatório das referências das três séries, para os 18 termos e conceitos mais referidos, revela-nos a seguinte sequência de ordenação decrescente: cuidar; pessoa; saúde, científico/ciência; bem-estar;

promover; doença; autocuidado; prevenção; profissão; arte; independência/dependência; conhecimento; objetivos; transição; humano; disciplina; autonomia (Figura 4).

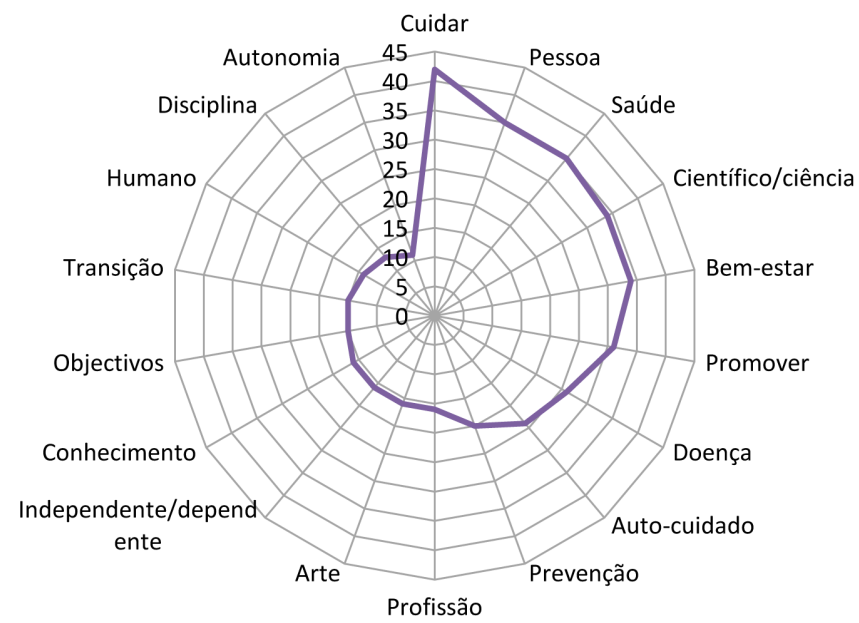

Figura 4. Frequências numericamente mais expressivas de termos e conceitos utilizados e apontados pelos estudantes de licenciatura e de mestrado no conjunto das três séries

Os termos e conceitos que emergiram neste estudo Fawcett (2005); Newman (1983); Kim (2010); Ordem foram comparados com os conceitos considerados dos Enfermeiros. Conselho de Enfermagem, (2002); como conceitos centrais pela literatura de enfermagem, Schumacher e Meleis (1994); Meleis e Trangenstein considerando os estudos de Yura e Torres (1975); (1994); Meleis (2012). Com esta comparação 
constatamos que os conceitos sociedade, enfermagem, paciente, paciente-enfermeiro, terapêuticas de enfermagem, não tiveram correspondência nos termos e conceitos nas três séries de dados deste estudo. $\mathrm{O}$ conceito cuidados de enfermagem/cuidar reuniu um total de 42 referências ocupando o primeiro lugar, seguido pelos conceitos pessoa e saúde ambos com um total de 35 referências. Em terceiro lugar, e não distanciado dos anteriores, o conceito de bem-estar com 34 referências. As transições surgem em quarto lugar,já com uma quebra significativa, 15 referências. De seguida e com menor expressão: quinto lugar, processo de enfermagem, com 7 referências; sexto lugar, prática, com 6 referências; sétimo lugar, facilitação, com 5 referências; e em oitavo lugar ambiente e interação com uma referência cada (Tabela 4).

\section{Tabela 4}

\section{Identificação dos termos e conceitos presente nas três séries com os conceitos centrais da literatura}

\begin{tabular}{|c|c|c|c|c|c|}
\hline Autores & Conceitos da literatura & IA & II B & II C & Totais \\
\hline $\begin{array}{l}\text { Yura e Torres, } 1975 \\
\text { OE, } 2002 \\
\text { Fawcett, } 2005\end{array}$ & $\begin{array}{l}\text { Pessoa } \\
\text { (ser humano) }\end{array}$ & 12 & 16 & 7 & 35 \\
\hline Yura e Torres, 1975 & Sociedade & & & & \\
\hline $\begin{array}{l}\text { Yura e Torres, } 1975 \\
\text { Newman, } 1983 \\
\text { OE, 2002 } \\
\text { Fawcett, } 2005 \\
\text { Meleis, } 2012\end{array}$ & Saúde & 12 & 17 & 6 & 35 \\
\hline $\begin{array}{l}\text { Yura e Torres, } 1975 \\
\text { Newman, } 1983 \\
\text { Fawcett, } 2005\end{array}$ & Enfermagem & & & & \\
\hline $\begin{array}{l}\text { Kim, } 2010 \\
\text { Newman, } 1983 \\
\text { OE, 2002 } \\
\text { Fawcett, } 2005 \\
\text { Meleis, } 2012\end{array}$ & Ambiente & 1 & & & 1 \\
\hline $\begin{array}{l}\text { Kim, } 2010 \\
\text { Newman, } 1983 \\
\text { Meleis, } 2012 \\
\end{array}$ & Paciente (cliente) & & & & \\
\hline OE, 2002 & Cuidados de Enfermagem (Cuidar) & 15 & 16 & 11 & 42 \\
\hline $\begin{array}{l}\text { Kim, } 2010 \\
\text { Newman, } 1983\end{array}$ & $\begin{array}{l}\text { Paciente-enfermeiro } \\
\text { (cliente-enfermeiro) }\end{array}$ & & & & \\
\hline Kim, 2010 & Prática & & 6 & & 6 \\
\hline Meleis, 2012 & Interação & 1 & & & 1 \\
\hline $\begin{array}{l}\text { Meleis e Trangenstein, } 1994 \\
\text { Schumacher e Meleis, } 1994 \\
\text { Meleis, } 2012\end{array}$ & Transição & & 8 & 7 & 15 \\
\hline Meleis, 2012 & Processo de Enfermagem & & 6 & 1 & 7 \\
\hline Meleis, 2012 & Terapêuticas de Enfermagem & & & & \\
\hline $\begin{array}{l}\text { Meleis e Trangenstein, } 1994 \\
\text { Meleis, } 2012\end{array}$ & Facilitação & & 4 & 1 & 5 \\
\hline $\begin{array}{l}\text { Meleis e Trangenstein, } 1994 \\
\text { Meleis, } 2012\end{array}$ & Bem-estar & 3 & 7 & 24 & 34 \\
\hline
\end{tabular}

O somatório dos termos e conceitos referenciados neste estudo (séries A B C) perfaz um total de 778. O total dos termos e conceitos que foi possível identificar com as propostas da literatura é de 171 (soma dos totais das séries A B C na Tabela 4) o que dá uma percentagem de identificação de 21,97. Pudemos comparar com os $9,67 \%$, valor percentual de conceitos identificados na série II C (24) com o total referido na mesma série (248). O que permite realçar a riqueza terminológica, ainda que relativa, de alguns termos usados para definir enfermagem em comparação com os conceitos significativos evocados pelos informantes. 


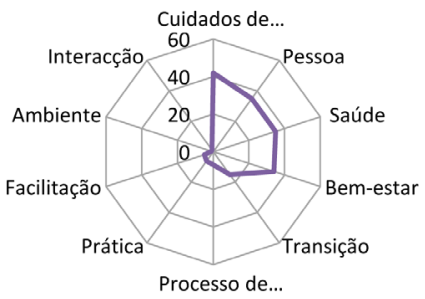

Figura 5. Identificação dos termos e conceitos presente nas três séries com os conceitos centrais da literatura, frequências mais expressivas.

Para o conjunto destas duas amostras e três séries de dados, os conceitos com centralidade (Figura 5) surgem por ordem decrescente da sequência: cuidados de enfermagem/cuidar, pessoa, saúde, bem-estar, com frequências altas; transição, com frequências intermédia; processo de enfermagem, prática, facilitação, ambiente, interação, com frequências mais baixas.

Interessou-nos ainda saber em que medida os termos das definições de enfermagem e os conceitos significativos se identificavam e alinhavam com os padrões de conhecimento que a literatura em enfermagem nos revela. Considerámos para o efeito dos padrões de conhecimento da literatura os trabalhos de Carper (2006); Shultz e Meleis
(1988); Moch (1990); White (2006); e Abreu (2008). Verificámos que 36 termos e conceitos se identificavam com o padrão de conhecimento empírico (ciência, científico, investigação); 16 com o padrão estético; 11 com o relacional; 7 com o processual; 5 com o cultural; 3 com o conceitual (teórico); e 2 com o contexto. Não encontrámos identidades para os padrões: conhecimento pessoal, ético, clínico, experimental, interpessoal, intuitivo e tácito. Embora se possa encontrar, numa análise menos taxativa, algumas similitudes entre alguns conceitos, nomeadamente, conhecimento pessoal com o conceito de si (II B), interpessoal com interação (I A) e mesmo para alguns conceitos como clínico e experimental (Tabela 5).

\section{TABELA 5}

Identificação dos termos e conceitos presente nas três séries com os padrões de conbecimento em enfermagem patentes na literatura

\begin{tabular}{|c|c|c|c|c|c|}
\hline Autores & Padrões do conhecimento & IA & II B & II C & Totais \\
\hline $\begin{array}{l}\text { Carper, } 2006 \\
\text { Shultz e Meleis, } 1988 \\
\text { White, } 2006 \\
\text { Abreu, } 2008\end{array}$ & Empírico (ciência/científico/investigação) & 14 & 14 & 8 & 36 \\
\hline $\begin{array}{l}\text { Carper, } 2006 \\
\text { White, } 2006 \\
\text { Abreu, } 2008 \\
\end{array}$ & Estético & 8 & 6 & 2 & 16 \\
\hline $\begin{array}{l}\text { Carper, } 2006 \\
\text { White, } 2006 \\
\text { Abreu, } 2008 \\
\end{array}$ & Conhecimento pessoal & & & & \\
\hline $\begin{array}{l}\text { Carper, } 2006 \\
\text { White, } 2006 \\
\text { Abreu, } 2008 \\
\end{array}$ & Ético & & & & \\
\hline Shultz e Meleis, 1988 & Clínico & & & & \\
\hline Shultz e Meleis, 1988 & Conceitual (teórico) & & 3 & & 3 \\
\hline Moch, 1990 & Experimental & & & & \\
\hline Moch, 1990 & Interpessoal & & & & \\
\hline Moch, 1990 & Intuitivo & & & & \\
\hline $\begin{array}{l}\text { White, } 2006 \\
\text { Abreu, } 2008\end{array}$ & Contexto & & 2 & & 2 \\
\hline Abreu, 2008 & Processual & & 6 & 1 & 7 \\
\hline Abreu, 2008 & Relacional & 4 & & 7 & 11 \\
\hline Abreu, 2008 & Cultural & & 3 & 2 & 5 \\
\hline Abreu, 2008 & Tácito & & & & \\
\hline
\end{tabular}




\section{Discussão}

Os estudantes da licenciatura em enfermagem utilizam mais termos para definir a enfermagem do que os estudantes do mestrado e mais termos para definir enfermagem do que conceitos com significado específico. Ainda assim existe, em ambos os grupos, uma forte dispersão de termos e conceitos reveladora de alguma dificuldade de precisão e objetividade na definição de enfermagem.

$\mathrm{Na}$ análise de conjunto, os termos e conceitos cuidar, pessoa, saúde e ciência são os mais utilizados e referidos. O termo cuidar é de facto o mais utilizado $\left(1^{\circ}\right.$ lugar na série I A; $2^{\circ}$ na série II B; $4^{\circ}$ na série II C; e $1^{0}$ na análise de conjunto). É ainda o que tem maior alinhamento, neste estudo, com os conceitos centrais definidos na literatura (42 referências).

O termo transição não é referido pelos estudantes que estão a iniciar o mestrado. Em relação aos alunos da licenciatura é utilizado oito vezes na definição de enfermagem (um modesto $6^{\circ}$ lugar), sendo ainda menos referido como conceito com significado $\left(7^{\circ}\right.$ lugar, 7 referências), e no conjunto das três séries situa-se em $15^{\circ}$ lugar. Constatamos que não há uma apropriação do termo e do conceito como seria expectável para o estado da arte.

$\mathrm{O}$ termo bem-estar nos estudantes a iniciar o mestrado ainda se encontra mais distanciado dos lugares cimeiros do que o termo transição, no entanto é já apontado como primeiro conceito pelos estudantes da licenciatura. No conjunto das três séries, o bemestar, encontra-se em $5^{\circ}$ lugar. 0 pensamento de Meleis e Trangenstein (1994) expresso no objetivo formulado para a enfermagem como a facilitação dos processos de transição tendo em vista o bem-estar, surge sem ser apropriado pelos estudantes a iniciar o mestrado. Mais nítida a não-apropriação no que toca ao bem-estar, existindo já algumas referências ao termo transiçã̃o. Os estudantes da licenciatura, de forma diferente, colocam o bem-estar como primeiro conceito, já não acontecendo o mesmo com o conceito transiçãa. Dos sete conceitos sugeridos por Meleis (2012) como centrais, só os conceitos paciente de enfermagem e terapêutica de enfermagem não são utilizados pelos estudantes. Estes privilegiam outros conceitos próximos desses como sejam pessoa e cuidar/cuidados.

Comparando os conceitos da literatura com os termos e conceitos utilizados pelos nossos informantes, está em primeiro lugar o cuidar, segue-se a pessoa, a saúde e o bem-estar. $\mathrm{O}$ ambiente e a sociedade são conceitos da literatura que não estão em linha com os conceitos e termos utilizados pelos estudantes. De referir que apenas 21,97\% das referências estão em consonância com os conceitos da literatura, se restringimos a análise aos conceitos com significado (II C) a percentagem cai para 9,67\%, o que nos leva a concluir uma deficiente apropriação dos conceitos existentes na literatura de teoria de enfermagem.

O padrão de conhecimento empírico reúne a maior frequência de termos e conceitos referidos, o que está em linha com a utilização do termo ciência ( $4^{\circ}$ lugar no conjunto, $2^{\mathrm{a}} \mathrm{e} 3^{\mathrm{a}}$ referência nas definições de enfermagem).

O padrão de conhecimento estético fica em segunda posição distanciada, menos de metade, do empírico, logo seguido do padrão relacional. O padrão ético não teve colagens, eventualmente por ser assumido de forma tão interiorizada que não houve necessidade dos estudantes o teorizarem. Pode ainda ter acontecido por outras razões, como as apresentadas no estudo de Correia e Costa (2012) com estudantes em final de curso, onde a dimensão imperativo moral ou ideal surge como a menos valorizada no significado atribuído ao cuidar.

Também não há a apropriação de termos e conceitos que revelem diretamente padrões de conhecimento pessoal, intuitivo e tácito. O termo e conceito de arte afastam-se dos primeiros lugares: $8^{\circ}$ lugar estudantes de mestrado, $12^{\circ}$ lugar para os estudantes da licenciatura, apenas com duas referências como conceito. Os estudantes facilmente percecionam e definem a enfermagem como ciência e menos como arte.

O termo saúde surge em $1^{0}$ lugar na licenciatura, $4^{0}$ lugar no mestrado, e com seis referências nos conceitos. Este termo tem ainda um bom alinhamento com os conceitos da literatura (trinta e cinco termos em alinhamento), apresentando-se em todas as séries mais central do que o conceito de bem-estar. O termo doença ocupa o $6^{\circ}$ lugar nos termos do mestrado, mas $2^{\circ}$ na licenciatura e $12^{\circ}$ nos conceitos.

$\mathrm{O}$ termo autocuidado surge como segundo conceito (II C) e apenas com cinco referências como termo definidor de enfermagem entre os estudantes da licenciatura, mas não é utilizado pelos estudantes a iniciar o mestrado. O termo autocuidado, comportando uma riqueza conceptual indiscutivel 
sob o ponto de vista teórico, encontra expressão na licenciatura mas não no mestrado. Isto leva-nos a pensar em menos apropriação de teorias e conceitos centrais nos estudantes a iniciar o mestrado e afastados do ensino formal há mais tempo.

\section{Conclusão}

Em síntese constatamos grande dispersão de termos e conceitos que revelam dificuldade dos estudantes na definição objetiva e precisa de enfermagem. O cuidar é o termo e conceito mais utilizado com maior apropriação; o pensamento de Meleis acerca da enfermagem, sobretudo em torno dos conceitos de transição e bem-estar não está apropriado pelos estudantes a iniciar o mestrado; os estudantes da licenciatura não dão centralidade ao termo transições mas valorizam com relevância o bem-estar. De uma forma geral, existe uma deficiente apropriação dos conceitos disciplinares de enfermagem disponibilizados pela literatura. Os estudantes da licenciatura e do mestrado facilmente percecionam e definem a enfermagem como ciência, valorizando o padrão empírico de conhecimento, e menos como arte, subalternizando outros padrões. Os estudantes a iniciar o mestrado, por afastados do ensino formal há mais tempo, apresentam uma menor apropriação de termos e conceitos disciplinares propostos pela teoria de enfermagem. Torna-se assim relevante que a formação inicial, pós-graduada e a contínua, em espaço formal ou em contexto de trabalho contribua e beneficie da sistematização da teoria produzida e seja expressa em linguagem disciplinar específica.

\section{Referências bibliográficas}

Abreu, W. C. (2008). Transições e contextos multiculturais. Coimbra, Portugal: Formasau.

Bardin, L. (1995). Análise de conteúdo. Lisboa, Portugal: Edições 70.

Carper, B. (2006). Fundamental patterns of knowing in nursing. In L. C. Andrist, P. K. Nicholas \& K. A. Wolf (Eds.), A history of nursing ideas (pp. 129-137). Sudbury, Canada: Jones and Bartlett Publishers.

Correia, M. A., \& Costa, M. A. (2012). Perceção do cuidar de um grupo de estudantes finalistas de enfermagem. Revista de Enfermagem Referência,3(8), 75-83. doi:10.12707/RIII11119
Fawcett, J. (2005). Contemporary nursing knowledge. Analysis and evaluation of nursing models and theories. Philadelphia, PA: F.A. Davis.

Fourez, G. (2008). A construção das ciências. As lógicas das invenções científicas. Lisboa, Portugal: Instituto Piaget.

Kim, H. S. (2010). The nature of theoretical thinking in nursing. New York, NY: Springer.

McEwen, M., \& Wills, E. (2009). Bases teóricas para enfermagem ( $2^{\mathrm{a}}$ ed.). São Paulo, Brasil: Artemed.

Medina, J. L. (1999). La pedagogía del cuidado. Saberes y prácticas en la formación universitária en enfermería. Barcelona, España: Laertes.

Meleis, A. (2012). Theoretical nursing: Development and progress (5th ed.). Pennsylvania, PA: Wolters Kluwer/Lippincott Williams \& Wilkins. doi:10.1016/0029-6554(94)90045-0

Meleis, A., \& Trangenstein, P. (1994). Facilitating transitions: Redefinition of a nursing mission. Nursing Outlook, 42(6), 255-259.

Moch, S. D. (1990). Personal knowing: Evolving research in nursing. Scholarly Inquiry for Nursing Practice, 4(2),155163.

Moya, J. L. M. (2005). Redescubrir el saber prático de la enfermería. El dificil equilibrio académico y profesional en la España «preeuropea» (III). Revista ROL de Enfermería, 28(7-8), 487-490.

Newman, M. (1983). The continuing revolution: A history of nursing science. In N. Chaska (Ed.), The nursing profession: A time to speak. New York, NY: McGraw-Hill.

Ordem dos Enfermeiros. Conselho de Enfermagem. (2002). Padrões de qualidade dos cuidados de enfermagem. Enquadramento conceptual, enunciados descritivos. Lisboa, Portugal: Autor.

Schumacher, K. L., \& Meleis, A. (1994). Transitions: A central concept in nursing. Image: Journal of Nursing Scholarship, 26(2),119-127. doi:10.1111/j.1547-5069.1994.tb00929.x

Schutz, P. R., \& Meleis, A. (1988). Nursing epistemology: Traditions, insights, questions. Image: Journal of Nursing Scholarship, 20(4), 217-221.

Waldow, V. (2009). Momento de cuidar: Momento de reflexão na ação. Revista Brasileira de Enfermagem, 62(1), 140-145. doi:10.1590/S0034-71672009000100022

White, J. (2006). Patterns of knowing: Review, critique, and update. In L. C. Andrist, P. K. Nicholas \& K. A. Wolf (Eds), A bistory of nursing ideas (pp. 139-150). Sudbury, Canada: Jones and Bartlett Publishers.

Yura, H., \& Torres, G. (1975). Today's conceptual frameworks with the baccalaureate nursing programs. In Faculty-curriculum development. Part III: Conceptual framework - Its meaning and function (pp. 17-75). New York, NY: National League for Nursing. 